\title{
Electronic structure study by means of X-ray spectroscopy and theoretical calculations of the "ferric star" single molecule magnet
}

\author{
A. F. Takác\&* and M. Neumann \\ Universität Osnabrück - Fachbereich Physik, D-49069 Osnabrück, Germany \\ A. V. Postnikov日 \\ Institut für Festkörperforschung, Forschungszentrum Jülich, D-52425 Jülich, \\ Germany and Russian Academy of Science - Ural Division, \\ S. Kowalewskoj 18, 620219 Yekaterinburg, Russia \\ K. Kuepper \\ Universität Osnabrück - Fachbereich Physik, D-49069 Osnabrück and Forschungszentrum Rossendorf, \\ Institut für Ionenstrahlphysik und Materialforschung, \\ Bautzner Landtrasse 128, 01328 Dresden, Germany \\ A. Scheurer, S. Sperner, and R. W. Saalfrank \\ Universität Erlangen-Nürnberg, Insitut für Organische Chemie, D-91054 Erlangen, Germany \\ K. C. Prince \\ Sincrotrone Trieste, Strada Statale 14, km 163.5, in Area Science Park, \\ I-34012 Basovizza (Trieste), Italy and INFM-TASC, \\ Laboratorio ELETTRA, I-34012 Basovizza (Trieste), Italy
}

(Dated: November 21, 2005)

\begin{abstract}
The electronic structure of the single molecule magnet system $\left\{M\left[\mathrm{Fe}\left(\mathrm{L}^{1}\right)_{2}\right]_{3}\right\} \cdot 4 \mathrm{CHCl}_{3}(M=\mathrm{Fe}$, $\left.\mathrm{Cr} ; \mathrm{L}^{1}=\mathrm{CH}_{3} \mathrm{~N}\left(\mathrm{CH}_{2} \mathrm{CH}_{2} \mathrm{O}\right)_{2}{ }^{2-}\right)$ has been studied using X-ray photoelectron spectroscopy, X-ray absorption spectroscopy, soft X-ray emission spectroscopy, as well as theoretical density functionalbased methods. There is good agreement between theoretical calculations and experimental data. The valence band mainly consists of three bands between $2 \mathrm{eV}$ and $30 \mathrm{eV}$. Both theory and experiments show that the top of the valence band is dominated by the hybridization between Fe $3 d$ and $\mathrm{O} 2 p$ bands. From the shape of the Fe $2 p$ spectra it is argued that Fe in the molecule is most likely in the $2+$ charge state. Its neighboring atoms $(\mathrm{O}, \mathrm{N})$ exhibit a magnetic polarisation yielding effective spin $S=5 / 2$ per iron atom, giving a high spin state molecule with a total $S=5$ effective spin for the case of $M=\mathrm{Fe}$.

PACS numbers: 75.50.Xx, 31.15.Ar, 33.20.Rm, 33.60.Fy
\end{abstract}

\section{INTRODUCTION}

In the quest for molecular magnets which might be useful for practical applications such as ultradense magnetic storage $e^{1}$, quantum computing ${ }^{2}$, or other interesting devices ${ }^{3}$, one finds at times molecules which combine structural beauty, promising chemistry for further development, non-trivial physics and practically interesting properties (intramolecular exchange interactions, magnetic anisotropy). Such new molecular materials often arise from metal-organic synthesis, which is the main driving force in studies of molecular magnets (see, e.g., the monograph by $\mathrm{Kahn}^{4}$, or Refs ${ }^{5,6,7}$ for recent reviews). The present work is devoted to one such system, known as "ferric star", which is simple enough to allow an accurate study of its electronic structure, yet far from trivial for what regards its chemical manipulation (crystallization with different ligands) and physical treatment (e.g., deposition on surfaces). The synthesis of magnetic molecules with the formula $\left\{M\left[\mathrm{Fe}\left(\mathrm{L}^{1}\right)_{2}\right]_{3}\right\} \cdot 4 \mathrm{CHCl}_{3}$, where $\mathrm{L}^{1} \equiv \mathrm{CH}_{3} \mathrm{~N}\left(\mathrm{CH}_{2} \mathrm{CH}_{2} \mathrm{O}\right)_{2}{ }^{2-}$ is an organic ligand and $M$ is $\mathrm{Fe}$ or $\mathrm{Cr}$, has been described by Saalfrank et $a l^{8}$ (compounds $\mathbf{1 1}$ and $\mathbf{1 2}$ of this paper). Previous basic magnetochemical investigations of the "ferric star" 9 indicate that the three spins $S=5 / 2$ of peripheral Fe ions at the star edges couple with an antiparallel orientation to the central ion, yielding (for $M=\mathrm{Fe}$ ) the magnetic ground state of $S=5$. In the present work we attempt to clarify the electronic structure, as it emerges from a combined spectroscopic study and first-principles calculations.

Specifically, we probe core-level excitations of the ferric star at the $L$ and other edges by several core spectroscopies, namely near-edge X-ray absorption spectroscopy (NEXAS), resonant inelastic X-ray scattering (RIXS) and X-ray photoemission spectroscopy (XPS). The electronic structure calculations were done from first principles within the density functional theory. The spectra give insight into the distribution of energy-resolved state densities within the valence band, which reveal the $M 3 d$ - O $2 p$ hybridization, and into charge states of transition metal (TM) atoms. The calculations give estimates of local magnetic moments, spatial distribution of the spin 

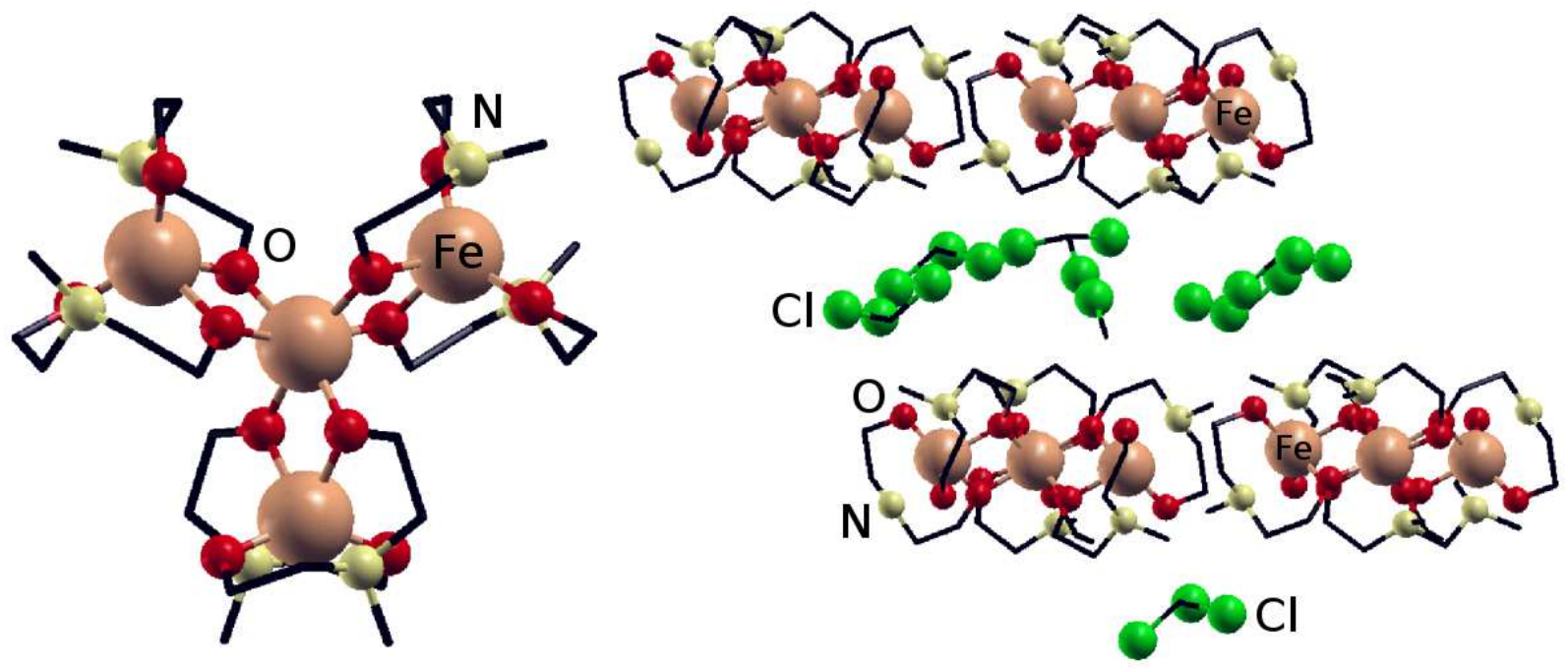

FIG. 1: Single "ferric star" molecule in the top view (left) and side view of the molecules packed in the crystal structure with chloroform (right). Hydrogen atoms are omitted, and carbon chains are shown as wireframe.

density, and of magnetic interaction parameters.

The paper is organized as follows. In Sec. II we summarize present knowledge of the crystal structure and basic magnetic properties. Sec. III gives technical details of the spectroscopy measurements, and of the firstprinciples electronic structure calculations. The discussion of results and comparison with the calculations is done in Sec. IV

\section{STRUCTURE AND MAGNETIC PROPERTIES}

The samples for our present spectroscopy study, as well as the crystallographic data used in electronic structure calculations, originate from the the Institute of Organic Chemistry of the University of Erlangen - Nürnberg 8 . The precise chemical formula of the compound in question is $\left\{M\left[\mathrm{Fe}\left(\mathrm{L}^{1}\right)_{2}\right]_{3}\right\} \cdot 4 \mathrm{CHCl}_{3}$, where $M$ is $\mathrm{Fe}$ or $\mathrm{Cr}$ in the present study. In the following we refer to these systems as $\mathrm{FeFe}_{3}$ and $\mathrm{CrFe}_{3}$-stars. The structure of a single molecule derived from crystal structure analysis can be seen in the left panel of Fig. 11 while the spatial packing of the molecules crystallized from chloroform is shown in the right panel. The central iron ion is linked via two $\mu_{2}-$ alkoxo bridges from each of the three terminal building blocks $\left[\mathrm{Fe}\left(\mathrm{L}^{1}\right)_{2}\right]^{-}$. The peripheral iron centers are octahedrally coordinated through two $\mathrm{N}^{-}, \mu_{1} \mathrm{O}_{-}^{-}$, and $\mu_{2} \mathrm{O}_{-}^{-}$ donors. All four iron ions are located in a plane, with $\mathrm{Fe}-\mathrm{Fe}-\mathrm{Fe}$ angles of about $120^{\circ}$. As known from the literature, similar types of ferric stars with the $\mathrm{FeFe}_{3}$ - framework can be synthesized using different ligands like dipivaloylmethane $(\mathrm{Hdpm})$ in combination with methanolate or 1,1,1 - tris(hydroxymethyl)ehane $\left(\mathrm{H}_{3}\right.$ thme), yielding respectively $\left[\mathrm{Fe}_{4}(\mathrm{OMe})_{6}(\mathrm{dpm})_{6}\right]^{\frac{9}{}}$ or $\left[\mathrm{Fe}_{4}(\text { thme })_{26}\right]^{10}$. The chemistry of $\left\{M\left[\mathrm{Fe}\left(\mathrm{L}^{1}\right)_{2}\right]_{3}\right\} \cdot 4 \mathrm{CHCl}_{3}$ has certain sim- ilarities with that of "ferric wheels" 11 , which have the same ligands and a similar (nearly octahedral) coordination of the Fe ions. A relation between the two systems is demonstrated by the fact that adding two equivalents of iron (III) chloride to $\left[M\left\{\mathrm{Fe}\left(\mathrm{L}^{1}\right)_{2}\right\}_{3}\right]$ produces the known "ferric wheel" with a metallacrown structure as the final product ${ }^{8}$. As in these latter compounds which we have studied earlier ${ }^{12.13}$, the spins $S=5 / 2$ of Fe ions tend to couple antiparallel. Contrary to "ferric wheels" with even numbers of Fe atoms, which have zero spin in the ground state, in the "ferric star" the antiparallel coupling of three outer Fe spins to the central one results in a net ground-state $\operatorname{spin} S=3 \times 5 / 2-5 / 2=5$. For the magnetic anisotropy, the effective Hamiltonian of which can be conventionally written in the form $\mathcal{H}=D S_{z}^{2}+E\left(S_{x}^{2}-S_{y}^{2}\right)$ in terms of the cumulative spin of the molecule, the axial parameter $D$ is $-0.20 \mathrm{~cm}^{-1}(-0.29 \mathrm{~K})$ from the high field electron spin resonance experiments 9.14 . The absolute value of $E$ (the sign may depend on the definition) was predicted by Kortus to be $0.064 \mathrm{~K}$ from a first-principle calculation $^{15}$, and later estimated as $0.056 \mathrm{~K}$ in experiments by Müller et al $l^{16}$.

It has been recently demonstrated that Fe in "ferric star" can be substituted by other $3 d$ ions. In the present work we study the spectroscopy of Cr-doped "stars". The accuracy of the amount and position of the substitution has not yet been unambiguously established. For ab initio calculations we assumed the substitution in the central position, in an otherwise unchanged geometry. A more systematic theoretical analysis of the electronic structure of "ferric wheels" with substitution by different ions in different positions will be reported elsewhere ${ }^{17}$. 


\section{EXPERIMENTAL AND CALCULATION DETAILS}

The XPS measurements were performed using a PHI Model 5600ci MultiTechnique system in the Dept. of Physics, University of Osnabrück. The $\mathrm{Al} K_{\alpha}$ radiation was monochromatized by a double focusing monochromator and the pressure during the measurements was typically $10^{-9} \mathrm{mbar}$. The total energy resolution, as determined at the Fermi level of a gold foil, was 0.3-0.4 $\mathrm{eV}$. The resolution of the electron energy analyzer was $80 \mathrm{meV}$. The samples were insulating, so charge neutrality on the surface was achieved using a low energy electron flood gun. The recording time of the presented spectra was less than $10 \mathrm{~h}$. The NEXAFS and RIXS measurements where carried out at the $\mathrm{BACH}$ beamline of the synchrotron radiation facility in Trieste, using the COMIXS spectrometer ${ }^{18,19}$.

Normal X-ray emission spectroscopy was performed also at the ROSA beamline at the BESSY II Synchrotron facility in Berlin, using the undulator based beamline ID12-2, and the rotatable spectrometer apparatus (ROSA) at BESSY II, Berlin. The excitation energies were set to $750 \mathrm{eV}$ for the $\mathrm{Fe} L$ edge, to $420 \mathrm{eV}$ for the N $K$ edge, and to $600 \mathrm{eV}$ for the $\mathrm{O} K$ edge. The overall resolution (beamline plus spectrometer) was set to around $1 \mathrm{eV}$.

For X-ray photoemission spectroscopy, emitted electron energies were calibrated to the $\mathrm{C} 1 s$ line from $\mathrm{CH}_{2}-$ $\mathrm{CH}_{2}-\mathrm{O}^{20}$. For $\mathrm{X}$-ray absorption the resolution was set to $0.3 \mathrm{eV}$ and for emission to $0.7 \mathrm{eV}$. The emitted photon energies at the iron edge were calibrated to the $\mathrm{Fe}$ metal $L_{\alpha}$ normal emission maximum at $705 \mathrm{eV}$ and at the oxygen $K$ edge to the emission from $\mathrm{MgO}$ at $525 \mathrm{eV}$. The pressure during the measurements was $10^{-9}$ mbar.

The X-ray photoelectron spectra were recorded of $\mathrm{Fe}$ $(3 s, 2 p), \mathrm{C} 1 s, \mathrm{O} 1 s$, and $\mathrm{N} 1 s$ core levels. The main peaks of the Fe3s spectra of the "ferric star" were fitted with a Voigt function 21 , by constraining the Gaussian width to a value of $0.6 \mathrm{eV}$. The background was simulated by a Tougaard function ${ }^{22}$, and subtracted from the spectra.

The electronic structure of the "ferric stars" was calculated from first principles within the density functional theory, using the calculation method and computer code Siesta $23,24,25$. The method uses normconserving pseudopotentials in combination with atomcentered strictly confined numerical basis functions ${ }^{26.27}$. The basis set included double- $\zeta$ functions with polarization orbitals added for $\mathrm{Fe}$ and $\mathrm{O}$. The treatment of exchange-correlation was done according to the generalized gradient approximation after Perdew-BurkeErnzerhof ${ }^{28}$.

The single molecular unit $\left\{M\left[\mathrm{Fe}\left(\mathrm{L}^{1}\right)_{2}\right]_{3}\right\}$ was put into a simulation cell of size $22 \times 22 \times 18 \AA$, over which the fast Fourier transform of the charge density has been done with the cutoff 260 Ry. This corresponds to $216 \times 216 \times 180$ divisions along the simulation cell edges, and was tested to be sufficient to obtain converged energy differences between different magnetic configurations. A discrete energy spectrum was broadened in the following figures with the halfwidth parameter of $0.1 \mathrm{eV}$ in order to get a continuous density of states (DOS). The calculation can be converged to both the ferrimagnetic configuration (the ground state) and the antiferromagnetic configuration (with the spin of one of the outer Fe atoms set parallel to the central one), which is $0.13 \mathrm{eV}$ higher in energy.

\section{ELECTRONIC STRUCTURE AND CHARGE STATE OF Fe}

We start with a discussion of electronic structure of the "ferric star" by comparing the spectroscopic data concerning the valence band and first-principles calculations. The XPS spectrum of the valence band is shown in Fig. 2 along with the element-selective $\mathrm{X}$-ray emission spectra of Fe, O, and N. The emission spectra in Fig. 2 correspond to normal non-resonant fluorescence 29 . Taking into account the binding energies of the corresponding core levels from the XPS $\left(710.76 \mathrm{eV}\right.$ for Fe $2 p_{3 / 2}, 531.96$ $\mathrm{eV}$ for $\mathrm{O} 1 s, 400.76 \mathrm{eV}$ for $\mathrm{N} 1 s)$, the emission spectra were brought to a common energy scale with the valenceband XPS, and they are plotted in Fig. 2 This helps to identify the origin of pronounced features in the valence band. The peak at $6 \mathrm{eV}$ binding energy is mostly due to $\mathrm{Fe} 3 d 4 s$ states, but the $\mathrm{O} 2 p$ states contribute in about the same region (from $\sim 7 \mathrm{eV}$ to higher binding energy). The N $2 p$ states are located significantly deeper, forming a broad band centered at about $9 \mathrm{eV}$ binding energy.

These experimental observations are consistent with the results of first-principle calculations, which produce the DOS (summed over both spin directions) as shown in Fig. 3 The top panel depicts the total DOS (summed over both spin directions) in the ground state. Zero energy separates occupied and vacant states. One finds sev-

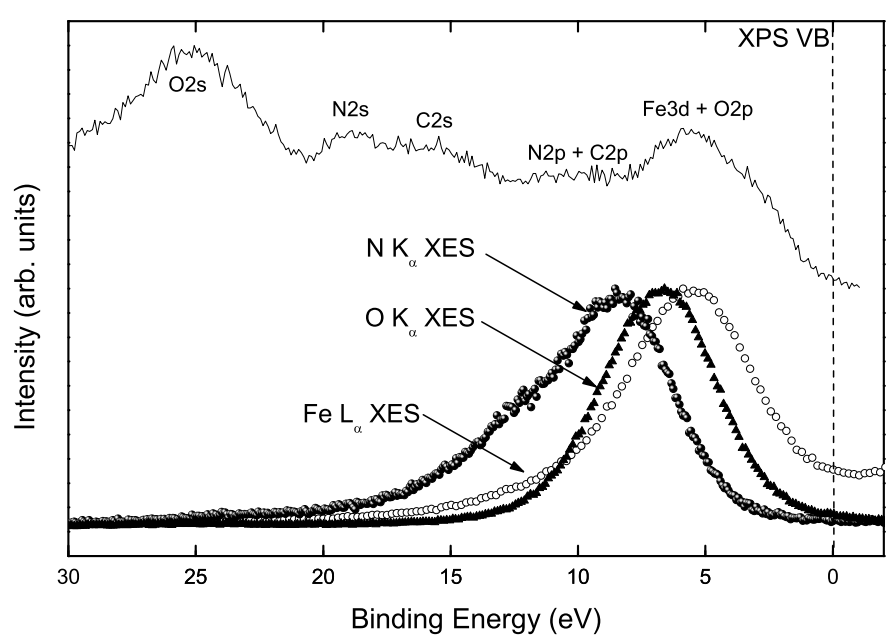

FIG. 2: Valence-band X-ray photoelectron spectrum of the Fe- "ferric star" and X-ray emission spectra of O, N, and Fe. 

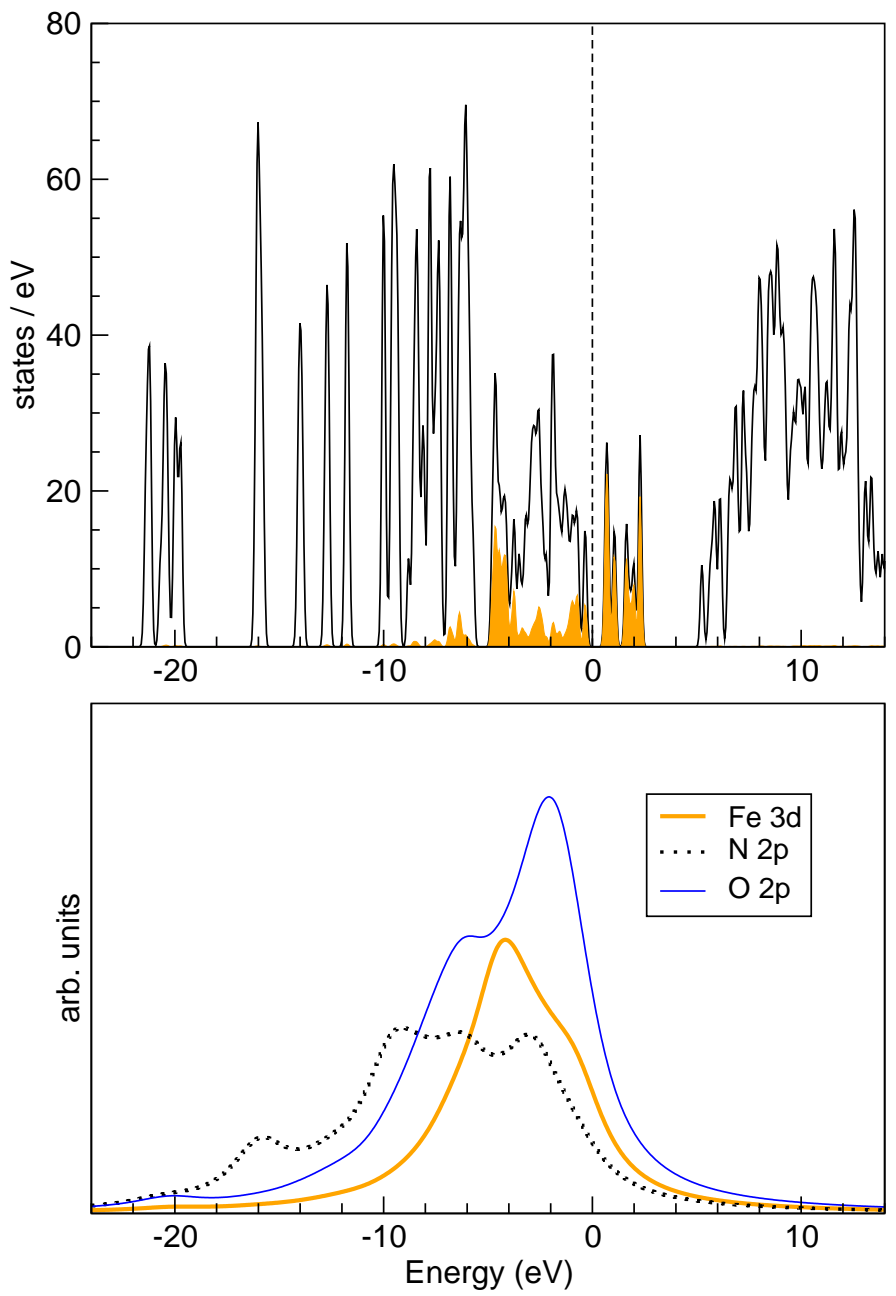

FIG. 3: Top panel: calculated total density of states of the Fe-"ferric star" and local Fe3d density of states (shaded area). Bottom panel: local densities of occupied $\mathrm{O} 2 p, \mathrm{Fe} 3 d$ and $\mathrm{N} 2 p$ states, additionally convoluted with a Lorentzian function of $1.6 \mathrm{eV}$ halfwidth. See text for details.

eral bands, which are derived from the $\mathrm{C}, \mathrm{N}$, and $\mathrm{O} 2 \mathrm{~s}$ and $2 p$ levels. The lowest compact group of bands around $-20 \mathrm{eV}$ is almost exclusively related to $\mathrm{O} 2 s$ states. The region around the "chemical potential" $(E=0)$ hosts Fe $3 d$ states (shown shaded in the figure), which are strongly spin-split (see the discussion below) and strongly hybridize with the $\mathrm{O} 2 p$ states. There is a "band gap" of $\approx 0.95 \mathrm{eV}$ between the highest occupied and the lowest unoccupied molecular orbitals, both being of mostly $\mathrm{Fe}$ $3 d-\mathrm{O} 2 p$ character in the majority-spin channel (marked spin-down in the following spin-resolved plot). The states with the energies from -16 to $-6 \mathrm{eV}$ are all a mixture of different orbitals forming covalent bonds and involving $\mathrm{C}$ $2 s, 2 p$ and $\mathrm{N} 2 s, 2 p$ states.

The bottom panel of Fig. 3 shows Fe $3 d, \mathrm{~N} 2 p$ and $\mathrm{O} 2 p$ contributions, summed up over atoms of the same kind in different positions in the molecule, and over both spin directions; moreover an additional broadening was intro- duced to yield a more straightforward comparison with the experimental XES of Fig. 21 Indeed these three partial DOS roughly span the states probed by the emission spectra, therefore the comparison is complicated by the lack of structure in the experimental spectra. One notices however a markedly lower energy and much larger width of the $\mathrm{N}$ spectrum as compared to other two, in both experiment and theory. The broadness of the $\mathrm{N}$ spectrum indicates the participation of $\mathrm{N} 2 p$ states in a number of molecular orbitals, overlapping with $\mathrm{C} 2 s, 2 p$ states throughout the molecule.

Spin-resolved DOS for the atoms in which the spin

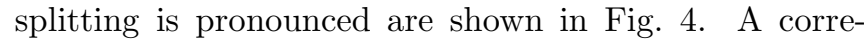
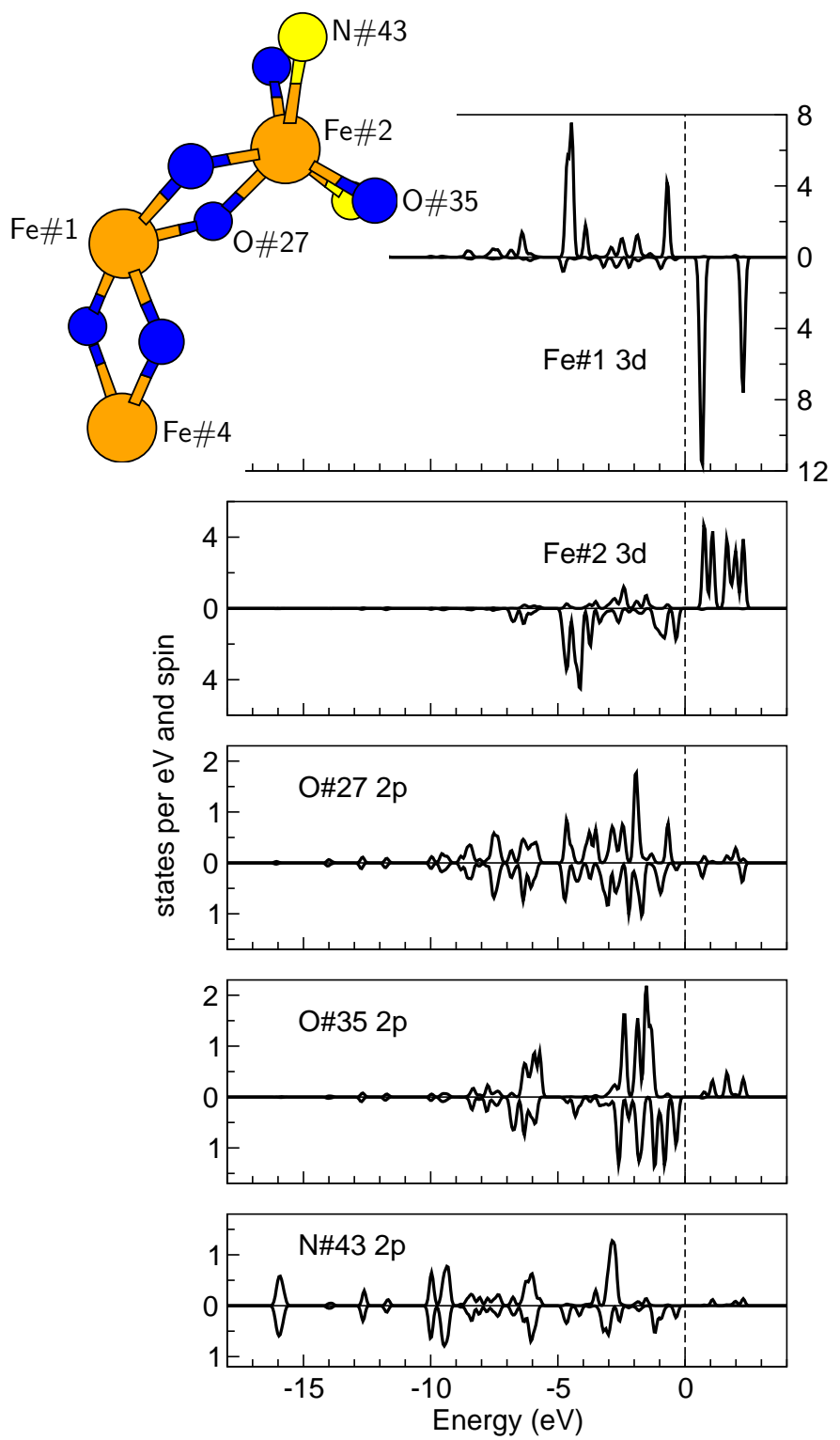

FIG. 4: Spin-resolved local densities of states of two inequivalent Fe sites and some their nearest neighbors. The numbering of atoms in the relevant fragment of "ferric star" is shown in the inset. 
sponding part of the molecule is shown in the inset, with the numbering of atoms. The central atom Fe\#1, which has the bridge oxygen atoms (\#27) as its only neighbors, carries a magnetic moment of $3.95 \mu_{\mathrm{B}}$. The outer Fe atoms (\#2) in the ground state are magnetized oppositely to the central one, with magnetic moments of -3.93 $\mu_{\mathrm{B}}$, and have a different environment, including two $\mathrm{N}$ atoms and two $\mathrm{O} \# 35$. The latter are markedly magnetized, to $-0.26 \mu_{\mathrm{B}}$. The bridge oxygen atoms have a negligible net magnetic moment, but a marked local spin density which changes sign along the path from Fe\#1 to $\mathrm{O} \# 27$ to $\mathrm{Fe} \# 2$. The induced magnetization at the oxygen atoms adds to the nominal spin moment associated with each Fe atom. The "local" moment of $\sim 4 \mu_{\mathrm{B}}$, which is associated with Fe3d states only, should be increased to $\sim 5 \mu_{\mathrm{B}}$ if one discusses a distributed magnetic moment, which in part resides over the ligands, and follows the magnetization flips of its central Fe atom if they occur. Hence, it is a "well-behaved" rigid moment in the sense that the Heisenberg model, or another model dealing with well defined spins, may be applied to it. These observations about the local DOS, induced magnetizations of ligands, and a delocalized but rigid spin moment associated with each Fe site are quite similar to what has been reported earlier for "ferric wheels", a chemically and structurally related class of molecular magnets 12.13 .

It is essential to emphasize that bulk techniques (magnetization, magnetic susceptibility) probe the effective spin value, however without addressing its localization. The issue of localization - whether the spin $S=5$ relates strictly to the Fe ion only or extends over ligands - is related to that of nominal valence. $\mathrm{Fe}^{3+}$ presumes the maximum-spin $3 d_{\uparrow}^{5} d_{\downarrow}^{0}$ configuration, whereas $\mathrm{Fe}^{2+}$ corresponds to the local magnetic moment of $4 \mu_{\mathrm{B}}$. Core-level spectroscopy, being an element-sensitive method, may permit one to distinguish between these two cases.

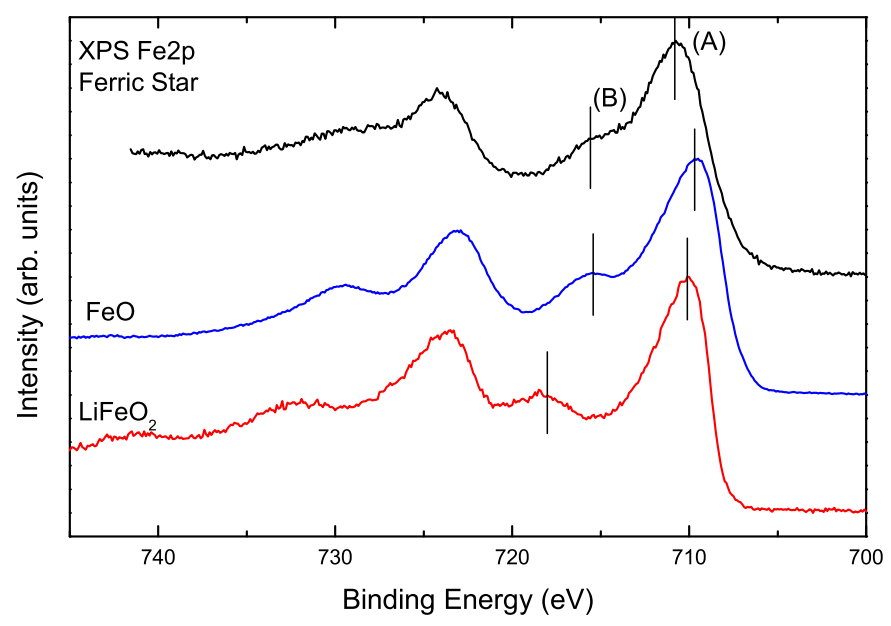

FIG. 5: Fe2p photoelectron spectrum of the Fe- "ferric star" in comparison with those of $\mathrm{FeO}$ (definitely $\mathrm{Fe}^{2+}$ ) and $\mathrm{LiFeO}_{2}$ (definitely $\mathrm{Fe}^{3+}$ ). The features (A) and (B) are discussed in the text.

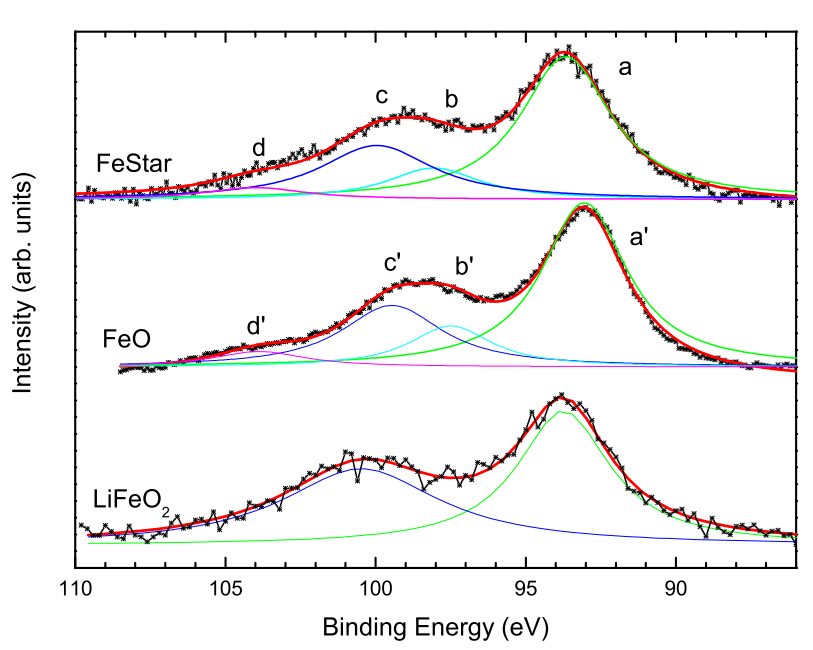

FIG. 6: Fe3s photoelectron spectrum of the Fe- "ferric star" in comparison with those of $\mathrm{FeO}$ (definitely $\mathrm{Fe}^{2+}$ ) and $\mathrm{LiFeO}_{2}$ $\left(\right.$ definitely $\mathrm{Fe}^{3+}$ ). The features (a), (b), (c) and (d) are discussed in the text.

Fig. 5 depicts the $2 p_{3 / 2}$ and $2 p_{1 / 2}$ photoelectron spectra of the "ferric star" along with those of two benchmark compounds, of well-known $\mathrm{Fe}^{2+}$ and $\mathrm{Fe}^{3+}$ valence: $\mathrm{FeO}$, similar to what was earlier discussed in ref 30 and $\mathrm{LiFeO}_{2}{ }^{31}$, respectively. Characteristic ligand-to-metal charge-transfer effects are present in the Fe $2 p$ spectra of the Fe "ferric star", as well as in the spectra of $\mathrm{FeO}$ and $\mathrm{LiFeO}_{2}$, yet there are some dissimilarities between these two reference systems. The relative positions and widths of the peaks due to the final state with charge transfer $2 p^{5} 3 d^{n+1} \underline{L}(\mathrm{~A})$ and that without charge transfer $2 p^{5} 3 d^{n} L$ (B) in the spectrum of "ferric star" is similar to that of the $\mathrm{FeO}$ which indicates the $\mathrm{Fe}^{2+}$ valence state.

The $3 s$ core-level photoelectron spectra of the "ferric star", again in comparison with those of $\mathrm{FeO}$ and $\mathrm{LiFeO}_{2}$, are shown in Fig. [6] The spectrum of the "ferric star" consists of two well separated peaks $(a)$ and $(c)$, according to whether the spin of the emitted $3 s$ core electron is parallel or antiparallel to that of the $3 d$ shell. Each of these peaks has a satellite denoted with $(b)$ and $(d)$, satellites which were assigned by Sangaletti and Pamigiani ${ }^{32}$ in the case of $\mathrm{FeO}$ to charge transfer excitations, with corresponding peaks labelled $a^{\prime}, b^{\prime}, c^{\prime}$ and $d^{\prime}$.

In $\mathrm{LiFeO}_{2}$ the charge transfer is not prominent, therefore the fitting was done using two peaks only. The splitting is proportional to the number of unpaired $d$ electrons and hence to total spin $S$; the predicted relative intensities are given by the relation $S /(S+1)^{33}$. The value of the Fe $3 s$ splitting in the case of the "ferric star" is about $5.10 \mathrm{eV}$, to be compared to $5.5 \mathrm{eV}$ in $\mathrm{FeO}$ and 6.5 $\mathrm{eV}$ in $\mathrm{LiFeO}_{2}$. We conclude that a nominal valence $\mathrm{Fe}^{2+}$ is more plausible in the "ferric star". 


\section{RESONANT X-RAY EMISSION IN PURE AND Cr-DOPED FERRIC STARS}

We turn now to the comparative analysis of pure and Cr-doped systems. The Cr substitution site is not unambiguously determined by experiment, whereas recent first-principle calculations presume that it must be the central one $e^{34}$. The valence-band XPS and Fe $L$-emission spectra are very similar in both systems, and Cr-related features cannot be resolved. However, certain differences were found when analyzing resonant X-ray emission, see Figs. 7 and 8 Panel (a) of Fig. 7 depicts the NEXAFS spectrum of the "ferric star" with $M=\mathrm{Fe}$, covering the Fe $L_{2,3}$ edges. The peak at about $709 \mathrm{eV}$ corresponds to the $\left(L_{3}\right)$ excitations from the Fe $2 p_{3 / 2}$ core state into the unoccupied $3 d$ band, and the second one at about 721 $\mathrm{eV}$ corresponds to those $\left(L_{2}\right)$ from the Fe $2 p_{1 / 2}$ state. The asymmetry seen in the first structure is very similar to that observed for $\mathrm{FeO}$, which has been explained in terms of many overlapping multiplets ${ }^{35}$. When comparing this two-peaked structure with the XPS, we note that here the $L_{2}-L_{3}$ splitting is $12.73 \mathrm{eV}$, i.e. less than the spin-orbit splitting of the $\mathrm{Fe} 2 p$ states determined by XPS $(14 \mathrm{eV})$. The reason for the discrepancy is that the both the XPS and absorption spectra consist of many

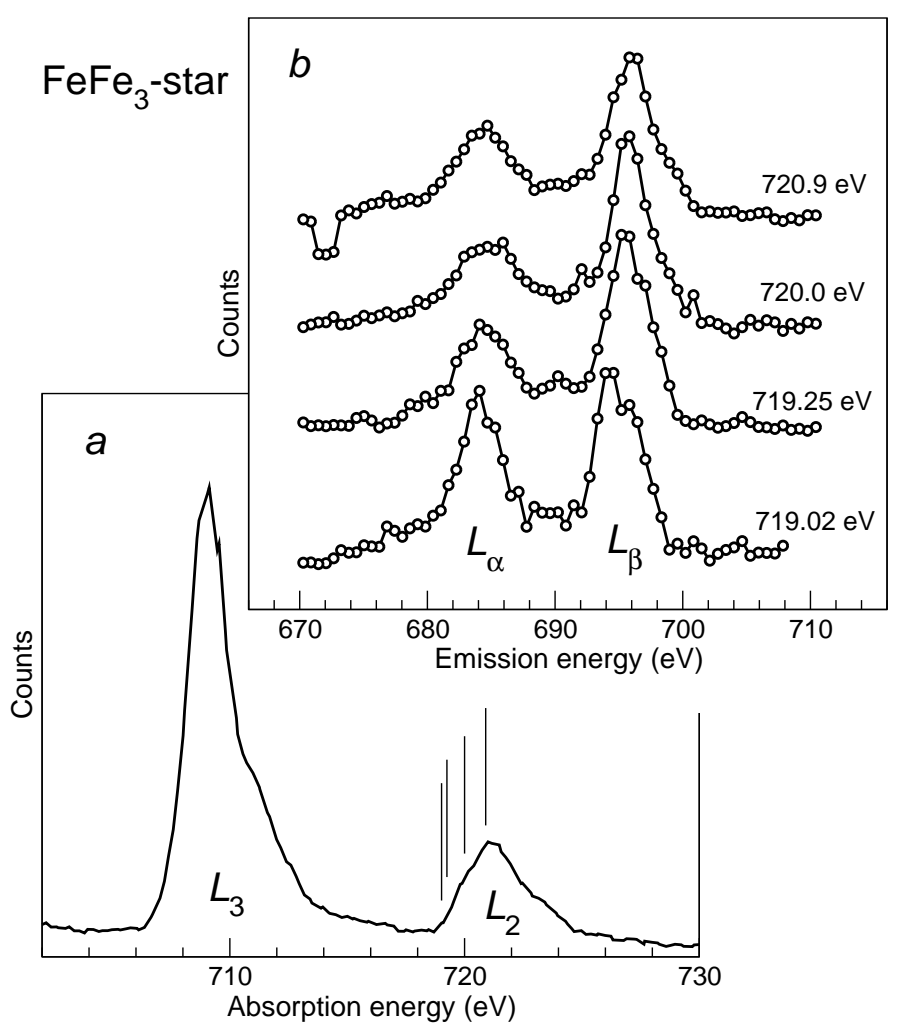

FIG. 7: Near-edge X-ray absorption Fe $L_{2,3}$ spectrum (a) and resonant inelastic X-ray Fe $L_{3}$ emission spectra (b) of the Fe"ferric star" for four selected excitation energies, which are also shown in the bottom panel as vertical bars. See text for details. multiplets, but these multiplets are different for the ionic (XPS) and neutral (absorption) final states, and therefore have different splittings. Panel (b) of Fig. 7 shows the X-ray emission spectra at the $L_{3}$ edge, obtained for four different incident photon energies: 719.02, 719.25, 720 , and $720.9 \mathrm{eV}$. Their positions at the threshold and on the top of the $L_{2}$ edge are indicated in the panel with the absorption spectrum.

In RIXS, a $2 p \rightarrow \mathrm{CB}$ (CB: conduction band) excitation is followed by a $\mathrm{VB} \rightarrow 2 p$ (VB: valence band) X-ray emission; both events are normally treated as a single joint process. As the excitation energy is gradually increased, on reaching the $L_{3}$ absorption threshold the $L_{\alpha}$ emission $\left(3 d \rightarrow 2 p_{3 / 2}\right)$ becomes possible. As the incident photon energy approaches the $L_{2}$ threshold, the $L_{\beta}$ emission $\left(3 d \rightarrow 2 p_{1 / 2}\right)$ appears as well. The $L_{\alpha}$ emission persists, as an off-resonance scattering process (creation and annihilation of a $2 p_{3 / 2}$ hole), but also possible by a CosterKronig process. In the latter, an initial $2 p_{1 / 2}$ hole is filled due to a radiationless $2 p_{3 / 2} \rightarrow 2 p_{1 / 2}$ transition, and the resulting $2 p_{3 / 2}$ hole is filled by $L_{\alpha}$ emission. These different mechanisms affect the $L_{\alpha} / L_{\beta}$ intensity ratio as a function of the excitation energy. We note that the $\mathrm{FeFe}_{3}$ and $\mathrm{CrFe}_{3}$ stars, in spite of many similarities in their $\mathrm{Fe} L$ spectra, behave markedly differently in what regards the

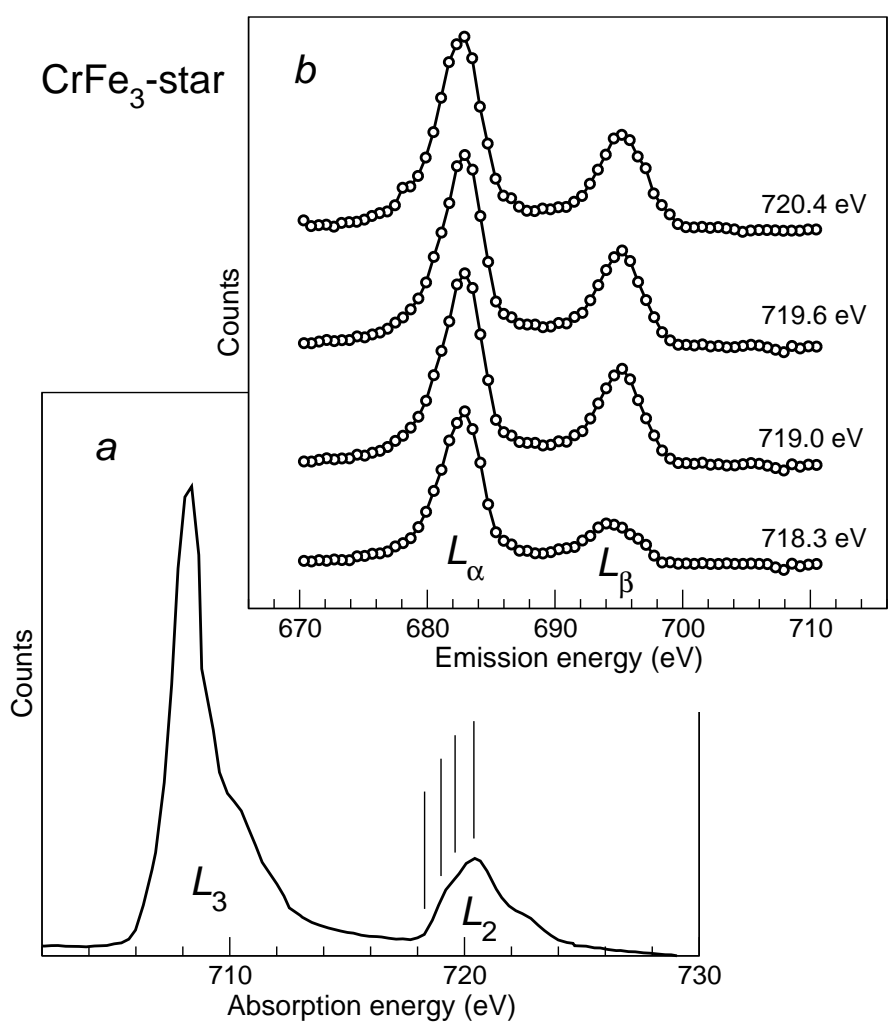

FIG. 8: Near-edge X-ray absorption Fe $L_{2,3}$ spectrum (a) and resonant inelastic X-ray Fe $L_{3}$ emission spectra (b) of the Cr"ferric star" for four selected excitation energies, which are also shown in the bottom panel as vertical bars. See text for details. 
$L_{\alpha} / L_{\beta}$ intensity ratio on passing through the $L_{2}$ resonance excitation. In Fig. 7(b) we observe that at the maximum of the absorption resonance, the $L_{\beta}$ intensity is higher than that of $L_{\alpha}$. We will see that this behavior is different from the $L_{\alpha} / L_{\beta}$ intensity ratio which occurs in the case of Cr substitution in the "ferric star".

Fig. 8 (a) depicts the NEXAFS at the Fe $L_{2,3}$ edge for the Cr-doped sample, and Fig. 8(b) - the X-ray emission spectra, taken at the resonance energies $718.3,719$, 719.6 and $720.4 \mathrm{eV}$, from the onset to the maximum of the $L_{2}$ absorption. The intensity of the $L_{\beta}$ emission first grows and then remains constant, relative to that of $L_{\alpha}$. The same behavior has been observed and discussed for the case of $\mathrm{FeO}$, in contrast to (non-magnetic) $\mathrm{FeS}_{2}$, by Prince et al. ${ }^{33}$. Another example of such behavior was reported for Mn $L$ RIXS spectra in Heusler alloys 36 . The interpretation of results in these papers related the $L_{\alpha} / L_{\beta}$ intensity to the high-spin state of Fe or Mn. More specifically, the importance of having an electronic structure with the majority-spin band occupied and the minority-spin band empty was emphasized. In this case a sequence of two processes, which may involve a Coster-Kronig transition as an intermediate step - an electron excitation into the $\mathrm{CB}$ and filling of the core hole by an electron from the VB - cannot occur except with the inversion of spin, which is a low-probability event. On the contrary, the electron excitation into the CB and an immediate fluorescence transition, which is a resonance process, remains allowed and explains the dominance of the $L_{\beta}$ emission on $L_{2}$ excitation.

It is known that Coster-Kronig transition rates are also affected by chemical environment. For example in the case of $\mathrm{Te}^{37}$, changing the chemical environment drastically from metallic to oxides to organometallic compounds produced a variation of $23 \%$ in the ratio of the widths of the $3 p_{3 / 2}$ to $3 p_{1 / 2}$ peaks. In the present case, the chemical environment is very similar so we expect small chemical effects. However the $L_{\alpha} / L_{\beta}$ branching ratio changes by 2 , which is a large effect simillar to that in 33 where the changes was nearly a factor of 3 . We therefore correlated the large change observed to changes in magnetic properties.

Fig. 4 makes clear that the local Fe DOS in the "ferric star" looks like that in a strong magnet, with the majority-spin band fully occupied, and only a small contribution (due to hybridization with $\mathrm{O} 2 p$ states) present in the occupied part of the minority-spin channel. Therefore the RIXS spectra in the $\mathrm{FeFe}_{3}$-star can be explained consistently with that in $\mathrm{FeO}$ or in Heusler alloys. The situation with the $\mathrm{CrFe}_{3}$-star, as is seen from the calculated local DOS shown in Fig. 9] is different: whereas the Fe local DOS (of the peripheral atoms, compare to Fig. (4) is identical to that in the $\mathrm{FeFe}_{3}$-star, the majorityspin states centered at the $\mathrm{Cr}$ site are not fully occupied. Moreover the lowest unoccupied states appear in the majority-spin channel. Therefore the absorptionemission processes involving the $\mathrm{Cr} 3 d$ states may happen without the inversion of $\operatorname{spin}^{38}$. However, we admit that

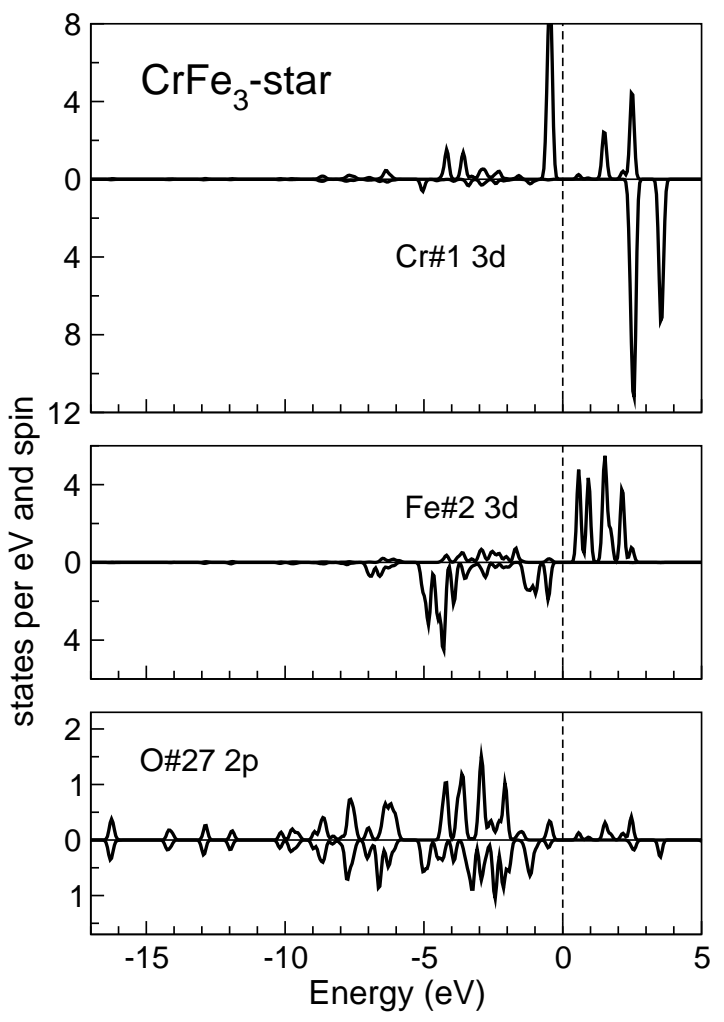

FIG. 9: Spin-resolved local densities of states of Cr, Fe and the bridging oxygen in the $\mathrm{CrFe}_{3}$-star, to be compared to Fig. 4

it is difficult to understand how this fact helps to reduce an apparent blocking of the Coster-Kronig transitions at the Fe site. Some clue may be provided by considering the bridging oxygen atoms (\#27 of Fig. 4): whereas in the $\mathrm{FeFe}_{3}$-star the magnetization density changes its sign exactly at the bridging $\mathrm{O}$, in the $\mathrm{CrFe}_{3}-$ star this oxygen atom is fully magnetized along with its Fe neighbor, and oppositely to $\mathrm{Cr}$. One can speculate that the matrix elements of the core-valence transitions, which include differently composed molecular orbitals in the valence band of $\mathrm{FeFe}_{3}$ - and $\mathrm{CrFe}_{3}$-stars, may affect relative weights of $L_{\alpha}$ and $L_{\beta}$ emission. This is however difficult to estimate without an explicit calculation of the RIXS process.

We can, with relative certainty, eliminate the following other hypothetical explanations of a different behavior in the $L_{\alpha} / L_{\beta}$ intensity ratio of the $\mathrm{CrFe}_{3}$-star. A possibility of a low-spin state is ruled out by the calculation result that in all trial spin-polarized calculations, the local magnetic moments remained essentially fixed to $\approx 3 \mu_{\mathrm{B}}$ at the $\mathrm{Cr}$ site and $\approx 4 \mu_{\mathrm{B}}$ at Fe sites. There is no way to "prepare" the system with a low-spin state of either $\mathrm{Cr}$, or $\mathrm{Fe}^{17}$. All inversions of individual local moments from the ground state, including the situation with the lowest total magnetic moment of $2 \mu_{\mathrm{B}}$ in the $\mathrm{Cr}_{\uparrow} \mathrm{Fe}_{\downarrow} \mathrm{Fe}_{\downarrow} \mathrm{Fe}_{\uparrow}$ star, preserve the "strong magnetism" at the Fe site and the partial emptiness of the majority-spin states at the $\mathrm{Cr}$ site, with strong similarities of their corresponding DOS 
to those shown in Figs. 4 and 9 Also the assumed $\mathrm{Cr}$ substitution in the peripheral position (highly energetically unfavorable, as was mentioned above) affected the magnetic moments and local DOS only slightly. An additional inclusion of on-site intraatomic Coulomb correlation beyond the conventional DFT treatment may affect the energy placements of $3 d$ levels in the $\mathrm{Cr}$ and Fe DOS, but it can only enhance the "strong magnetism" at the $\mathrm{Fe}$ site rather than ease it, in the presence of $\mathrm{Cr}$ dopant.

Therefore the understanding of $L_{\alpha} / L_{\beta}$ behavior in the $\mathrm{CrFe}_{3}$-star is incomplete on the basis of DFT calculations, whereas an explanation of the behavior in $\mathrm{FeFe}_{3}-$ star seems rather obvious, and consistent with the observed trend ${ }^{33}$ for $\mathrm{FeO}$, as compared to $\mathrm{FeS}_{2}$. We can only emphasize the observed difference in RIXS spectra of $\mathrm{FeFe}_{3}-$ and $\mathrm{CrFe}_{3}$-stars as an experimental finding, which might stimulate additional research on these systems.

\section{CONCLUSIONS}

We have reported photoemission and photoabsorption spectra of $\left\{M\left[\mathrm{Fe}\left(\mathrm{L}^{1}\right)_{2}\right]_{3}\right\} \cdot 4 \mathrm{CHCl}_{3}(M=\mathrm{Fe}, \mathrm{Cr})$ in comparison with first-principles electronic structure calculations. First we determined the charge state of Fe in these "ferric stars", in view of an existing controversy between the expected nominal chemical valence (Fe III), from one side, and the calculated value of the Fe local magnetic moment $\left(4 \mu_{\mathrm{B}}\right)$, that would imply a bivalent iron, from the other side. On the basis of a comparison of the Fe $2 p$ and $3 s$ photoelectron spectra with those of reference compounds, the charge state of $\mathrm{Fe}$ in the $\mathrm{FeFe}_{3}$-star was argued to be $2^{+}$. The valence-band X-ray photoelectron spectrum and the X-ray $\mathrm{Fe} L, \mathrm{O}$ and $\mathrm{N} K$ emission spectra show little structure but are otherwise in agreement with DFT calculations. In the $\mathrm{FeFe}_{3}-$ and $\mathrm{CrFe}_{3}$-stars, the $L_{\alpha} / L_{\beta}$ intensity ratio in resonant X-ray emission, varies according to the excitation energy on going through the Fe $L_{2}$ threshold. The trend observed in the $\mathrm{FeFe}_{3}$-star is similar to that earlier reported by Yablonskikh et al. and Prince et al., and believed to be a signature of "highspin" structures, or more precisely of strong magnetic systems, like e.g. FeO or Heusler alloys. The explanation of this behavior is consistent with the assumption that the Coster-Kronig process probability is suppressed in strong magnets. The DFT calculation does indeed support an assumption of such strong magnetic behavior in the $\mathrm{FeFe}_{3}$-star. However, the $\mathrm{CrFe}_{3}$-star does not show such a trend in the $L_{\alpha} / L_{\beta}$ intensity, even though the local electronic structure at the Fe site remains largely the same as in the $\mathrm{FeFe}_{3}$-star, and the loss of strong magnetic character appears confined to the $\mathrm{Cr}$ center.

\section{Acknowledgments}

AVP, AS, and SS gratefully acknowledge the financial support by the Deutsche Forschungsgemeinschaft (SPP 1137 'Molecular Magnets'), AT and KK acknowledge financial support of the $\mathrm{PhD}$ program of Lower Saxony (Germany). Many enlightening discussions with P. Müller, R. Fink and S. Blügel are appreciated. We thank J. Kortus for making available for comparison the unpublished results of his ab initio calculations on the ferric stars, which have been done prior to those of the present work. We thank the beamline scientists from the BACH beamline (ELETTRA) for the overall excellent support which they provided.
* Electronic address: altakacs@uni-osnabrueck.de

$\dagger$ Electronic address: apostnik@uos.de

1 M. Verdaguer, A. Bleuzen, C. Train, R. Garde, F. Fabrizi de Biani, and C. Desplanches, Phil. Trans. R. Soc. Lond. A 357, 2959 (1999).

2 M. N. Leuenberger and D. Loss, Nature 410, 789 (2001).

3 S.-i. Ohkoshi and K. Hashimoto, The Electrochemical Society Interface 11, 34 (2002).

4 O. Kahn, Molecular Magnetism (John Wiley \& Sons, Singapore, 1993), ISBN 0-471-18838-7.

5 W. Linert and M. Verdaguer, eds., Molecular Magnets (Springer-Verlag, Wien, 2003), ISBN ISBN: 3-211-838910, special Edition of Monatshefte für Chemie/Chemical Monthly, Vol. 134, No. 2.

6 J. S. Miller and A. J. Epstein, MRS Bulletin 25, 21 (2000).

7 S. J. Blundell and F. L. Pratt, J. Phys.: Condens. Matter 16, R771 (2004).

8 R. W. Saalfrank, I. Bernt, M. M. Chowdhry, F. Hampel, and G. B. M. Vaughan, Chem. Eur. J. 7, 2765 (2001).

9 A. L. Barra, A. Caneschi, A. Cornia, F. Fabrizi de Biani, D. Gatteschi, C. Sangregorio, R. Sessoli, and L. Sorace, J. Am. Chem. Soc. 121, 5302 (1999).
10 A. Cornia, A. C. Fabretti, P. Garrisi, C. Mortalo, D. Bonacchi, D. Gatteschi, R. Sessoli, L. Sorace, W.Wernsdorfer, and A. L. Barra, Angewandte Chemie International Edition 43, 1136 (2004).

11 R. W. Saalfrank, I. Bernt, E. Uller, and F. Hampel, Angewandte Chemie - International Edition 36, 2482 (1997).

12 A. V. Postnikov, S. G. Chiuzbăian, M. Neumann, and S. Blügel, J. Phys. Chem. Solids 65, 813 (2004).

13 A. V. Postnikov, J. Kortus, and S. Blügel, Molecular Physics Reports 38, 56 (2003).

14 D. Gatteschi, R. Sessoli, and A. Cornia, Chem. Commun. p. 725 (2000).

15 J. Kortus (unpublished).

16 R. Koch, S. Schromm, H. Rupp, and P. Müller, private communication.

17 A. V. Postnikov and S. Blügel, to be published.

18 M. Zangrando, M. Finazzi, G. Polucci, G. Comelli, B. Diciacco, R. P. Walker, D. Cocco, and F. Parmigiani, Rev. Sci. Instrum. 72, 1313 (2001).

19 D. Cocco, M. Matteucci, K. Prince, and M. Zangrando, Proceedings SPIE 4506 (2001).

20 G. Beamson and D. Briggs, eds., High Resolution XPS of 
Organic Polymers: The Scienta ESCA300 Database (John Wiley \& Sons, Chichester, 1992).

21 S. Doniach and M. Ŝnjić, Journal of Physics C 3, 285 (1970).

22 S. Tougaard, Surface and Interface Analysis 11, 453 (1988).

23 P. Ordejón, E. Artacho, and J. M. Soler, Phys. Rev. B 53, R10441 (1996)

24 J. M. Soler, E. Artacho, J. D. Gale, A. García, J. Junquera, P. Ordejón, and D. Sánchez-Portal, J. Phys.: Condens. Matter 14, 2745 (2002).

25 URL http://www . uam.es/siesta

26 D. Sánchez-Portal, E. Artacho, and J. M. Soler, J. Phys.: Condens. Matter 8, 3859 (1996).

27 J. Junquera, Ó. Paz, D. Sánchez-Portal, and E. Artacho, Phys. Rev. B 64, 235111 (2001).

28 J. P. Perdew, K. Burke, and M. Ernzerhof, Phys. Rev. Lett. 77, 3865 (1996).

${ }^{29}$ R. Szargan, K.-H. Hallmeier, R. Hesse, A. Kopczynski, S. A. Krasnikov, L. Zhang, T. Chassé, O. Fuchs, C. Heske, and E. Umbach, X-ray fluorescence spectroscopy at U41-PGM by means of ROSA-present status and results, BESSY Annual Report 2001.

30 B. Mayer, S. Uhlenbrock, and M. Neumann, J. Electron. Spectrosc. Relat. Phenom 81, 63 (1996).
31 V. R. Galakhov, A. I. Poteryaev, E. Z. Kurmaev, V. I. Anisimov, S. Bartkowski, M. Neumann, Z. W. Lu, B. M. Klein, and T.-R. Zhao, Phys. Rev. B 56, 4584 (1997).

32 F. Parmigiani and L. Sangaletti, J. Electron Spectrosc. Relat. Phenom. 98-99, 287 (1999).

${ }^{33}$ K. C. Prince, M. Matteucci, K. Kuepper, S. G. Chiuzbâian, S. Bartkowski, and M. Neumann, Phys. Rev. B 71, 085102 (2005).

34 According to our recent calculations, the central position of substitutional $\mathrm{Cr}$ is by $1 \mathrm{eV} /$ molecule more favorable than the peripherical one.

35 J. P. Crocombette, M. Pollak, F. Jollet, N. Thromat, and M. Gautier-Soyer, Phys. Rev. B 52, 3143 (1995).

36 M. V. Yablonskikh, Y. M. Yarmoshenko, V. I. Grebennikov, E. Z. Kurmaev, S. M. Butorin, L.-C. Duda, J. Nordgren, S. Plogmann, and M. Neumann, Phys. Rev. B 63, 235117 (2001).

37 M. K. Bahl, R. L. Watson, and K. J. Irgolic, J. Phys. Chem. 66, 5526 (1977).

38 We emphasize that the occupation of Fe or Cr states in the $\mathrm{FeCr}_{3}$ star remains qualitatively the same also for other possible configurations: when the $\mathrm{Cr}$ atom substitutes a peripheral site, or with different setting of magnetization directions over $\mathrm{Fe}, \mathrm{Cr}$ atoms. 